\title{
Water of Hydration Dynamics in Minerals Gypsum and Bassanite: Ultrafast 2D IR Spectroscopy of Rocks
}

\author{
Chang Yan, Jun Nishida, Rongfeng Yuan, Michael D. Fayer*
}

Department of Chemistry, Stanford University, Stanford, California 94305

*fayer@stanford.edu

\section{Supporting Information}

\section{X-ray diffraction measurements.}

The powder X-ray diffraction (XRD) measurements of gypsum and bassanite were performed on a PANalytical X'Pert instrument with $\mathrm{Cu} \mathrm{K \alpha}$ line. The diffraction patterns are shown in Figure S1. The gypsum pattern matches the record of JCPDS 01-070-0982. The bassanite pattern matches the record of JCPDS 00-041-0224.

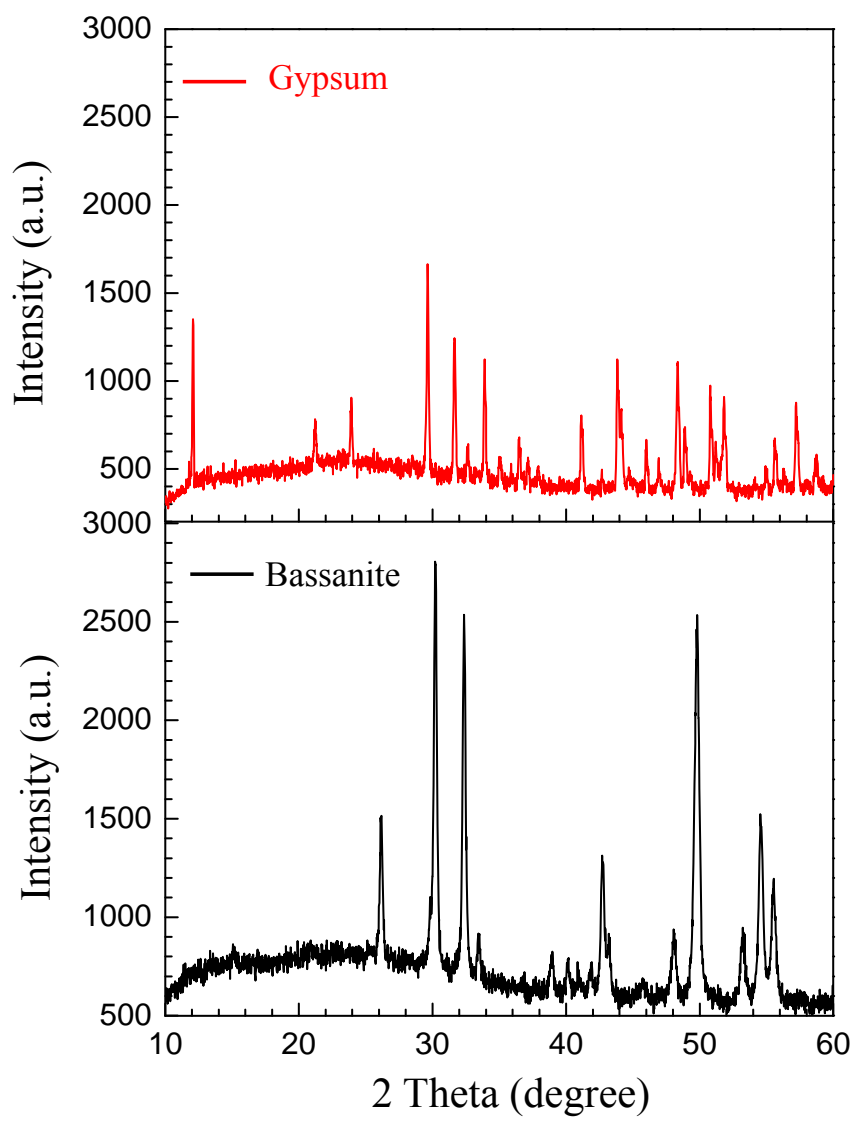


Figure S1. XRD powder patterns of gypsum and bassanite.

\section{Thermogravimetric analysis measurements.}

The thermogravimetric analysis (TGA) measurements of gypsum and bassanite were performed on a TGA Q500 analyzer produced by TA Instruments. The TGA curves of anhydrous $\mathrm{CaSO}_{4}$ (purchased from Sigma-Aldrich) and bassanite were shown in Figure $\mathrm{S} 2$. The weight loss of anhydrous $\mathrm{CaSO}_{4}$ was attributed to adsorbed volatile components from air and was subtracted as the background from the weight loss of bassanite. The weight loss of bassanite after subtraction was $6.5 \%$, corresponding to a chemical formula of $\mathrm{CaSO}_{4} \cdot 0.53 \mathrm{H}_{2} \mathrm{O}$.

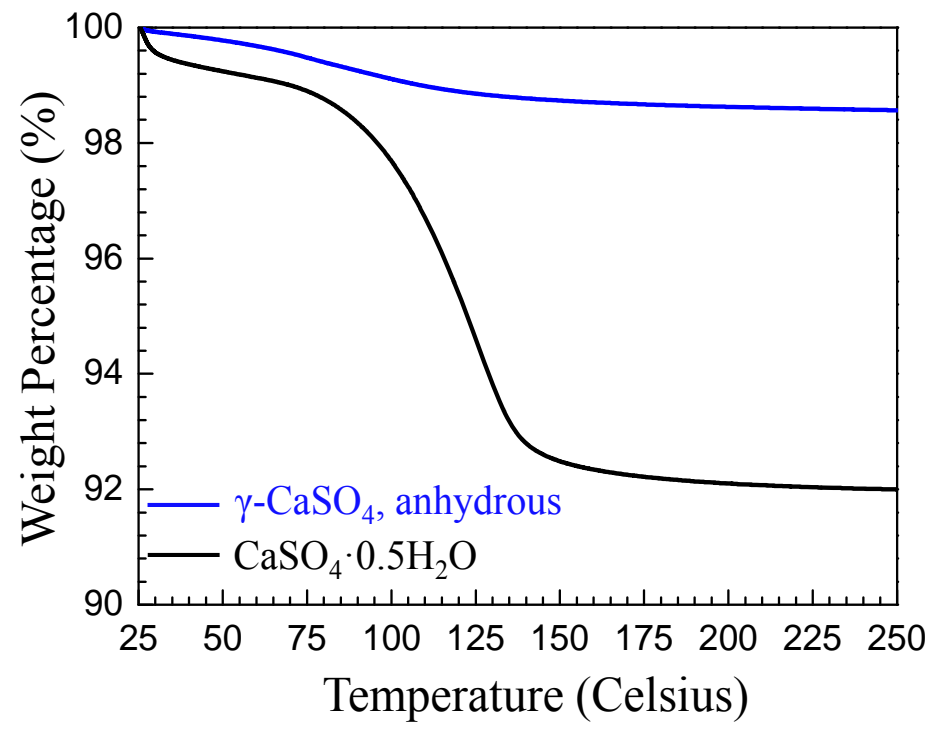

Figure S2. TGA curves of anhydrous $\mathrm{CaSO}_{4}$ and bassanite.

\section{FT-IR spectrum of bassanite exposed to moisture in air.}


The FT-IR spectrum of bassanite left open to air for 2 days is shown in Figure S3 (A). As a result of the channel structure in bassanite, moisture in air can permeate inside bassanite and exchange with the stoichiometric water molecules. This led to a loss of deuterium content and a decrease in the peak area of OD stretch band.

The line shape can be reproduced with a model of three Gaussians. As discussed in the main text, the line shape of bassanite prior to being exposed to air can also be fitted well with three Gaussian components. The peak widths and center positions of the Gaussians are nearly identical before and after exposing to air for 2 days. However, the relative amplitude of the magenta component significantly grew after the moisture attacked the sample. In Figure S3 (B), the normalized spectra clearly show that the tail on the red side became more prominent for bassanite exposed to moisture in air. Before exposing to air, the relative amplitude of the three components are: $17.2 \%$ (magenta), $79.9 \%$ (dark green), and $2.9 \%$ (blue). After exposing to air, the relative amplitude of the three components are: $26.2 \%$ (magenta), $70.1 \%$ (dark green), and $3.7 \%$ (blue).

Therefore, the magenta component at $2626 \mathrm{~cm}^{-1}$ was assigned to HOD molecules residing in channels deformed by additional non-stoichiometric water from moisture. The red-shifted peak position could be caused by a reduced O-O distance between sulfate ions and HOD molecules in defect channels or by additional hydrogen bonds formed between the stoichiometric $\mathrm{HOD}$ molecules and the additional $\mathrm{H}_{2} \mathrm{O}$ molecules 
picked up from moisture in the air.

A.

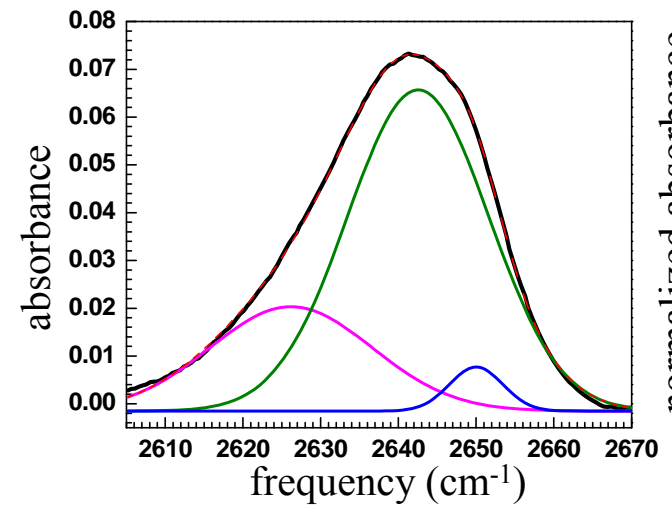

B.

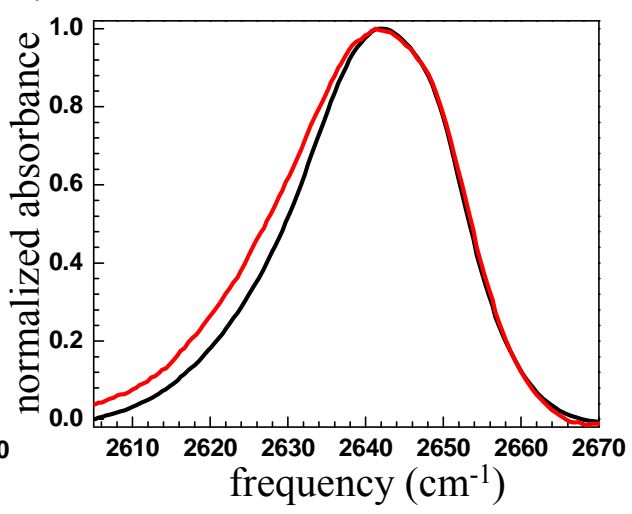

Figure S3. A. FT-IR spectrum of bassanite left in open air for 2 days. The red dashed line on top of the experimental result (black line) is the fitting line using three Gaussian components (magenta, dark green, and blue curves). B. Normalized spectra of bassanite stored in dry box (black line) versus bassanite left in open air for 2 days (red line).

\section{Temperature-dependent FT-IR spectra of bassanite.}

In Figure S4 we presented the comparison of bassanite's FT-IR spectra measured at $298 \mathrm{~K}$ and $348 \mathrm{~K}$. As the temperature increases, the relative ratio between the minor subensemble (blue line) and the major subensemble (dark green line) decreases. At $298 \mathrm{~K}$, the minor subensemble has $3.6 \%$ of the area of the major subensemble. At 333 $\mathrm{K}$, the minor subensemble has $2.3 \%$ of the major subensemble's area. At $348 \mathrm{~K}$, the percentage further drops to $1.8 \%$. The temperature-dependent FT-IR spectra suggest that the minor subensemble is in thermal equilibrium with the major subensemble. In Figure S5, we plotted the peak area ratios between the major and minor subensembles calculated from temperature-dependent FT-IR data. The slope of the fitting line multiplied by $\mathrm{R}=8.314 \mathrm{~J} / \mathrm{K}$ gives the enthalpy difference between the minor and major subensembles: $\Delta H=-12.0 \pm 0.2 \mathrm{~kJ} / \mathrm{mol}$. 
A.

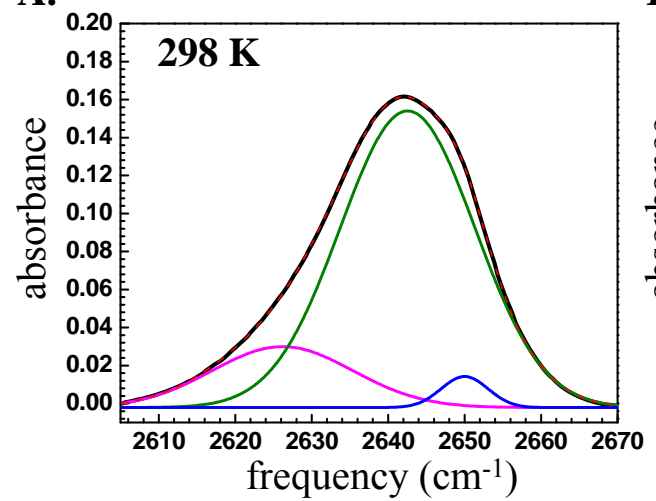

B.

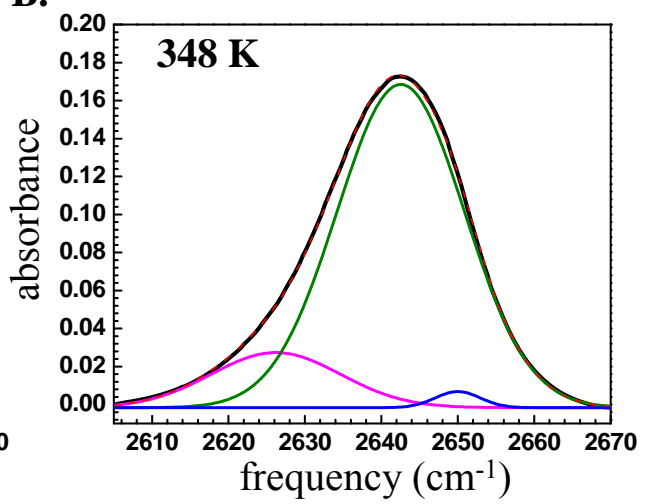

Figure S4. FT-IR spectrum of bassanite measured at $298 \mathrm{~K}$ (A) and $348 \mathrm{~K}$ (B). The red dashed line on top of the experimental result (black line) is the fitting line using three Gaussian components (magenta, dark green, and blue curves).

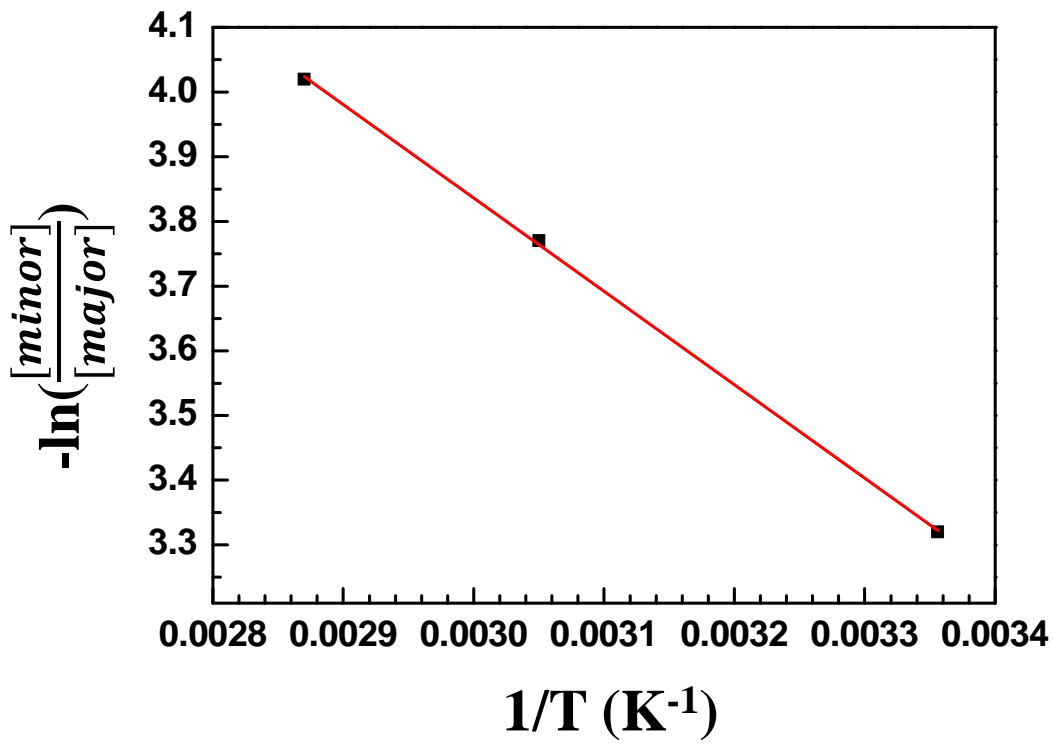

Figure S5. The FT-IR peak area ratios between the minor and major subensembles plotted versus the inverse of measurement temperatures. 